\title{
Diaphragm pacing and motor neurone disease: lessons for all?
}

\author{
Mark W. Elliott
}

Affiliation: Consultant Respiratory Physician, Dept of Respiratory Medicine, Sleep and Non-invasive Ventilation Service, St James University Hospital, Leeds, UK.

Correspondence: Mark W. Elliott, Dept of Respiratory Medicine, Sleep and Non-invasive Ventilation Service, St James University Hospital, Beckett Street, Leeds LS9 7TF, UK. E-mail: mark.elliott2anhs.net

@ERSpublications

Respiratory physicians and neurologists should be cognisant of the respiratory complications of motor neurone disease http://ow.ly/TIxe5

Respiratory muscle involvement is inevitable in motor neurone disease (MND) and most patients will die a respiratory death. Noninvasive ventilation (NIV) has been shown to prolong life and improve quality of life [1]. It is therefore important to identify patients who should be offered NIV at an early stage, as a delay may result in presentation with catastrophic respiratory failure, requiring endotracheal intubation, which almost always results in an unplanned tracheostomy. It used to be considered that respiratory muscle involvement was only a feature of advanced disease, but this is not correct; if looked for there is evidence of respiratory muscle weakness in most patients by the time the diagnosis is made [2]. In a small number, the diagnosis is made when the patient presents with hypercapnic respiratory failure.

The neurologist should include an assessment of respiratory muscle strength and ventilatory function when MND is diagnosed. The respiratory physician should consider neuromuscular disease, including MND, as part of the differential diagnosis when a patient presents with hypercapnic respiratory failure of unknown aetiology. The key factors for each specialty to consider are summarised in table 1.

The assessment of respiratory muscle strength is difficult in patients with bulbar involvement because they are usually unable to cooperate with tests of respiratory function. Assessment will need to be based more upon symptoms and clinical examination (reduced chest expansion) with early recourse to arterial blood gas analysis and overnight monitoring. It is noteworthy that in the trial of BouRKe et al. [1], in which patients were randomised on the basis of volitional tests of respiratory muscle function, the survival in the control group was comparable to that in the NIV group in nonbulbar patients. This indicates that volitional tests overestimate the severity of the respiratory muscle weakness.

Although overall NIV has been a significant step forward in the management of patients with MND, a significant proportion of patients, particularly those with bulbar involvement, do not tolerate it at all or tolerate it only poorly [5]. Any intervention, therefore, that delays or even obviates the need for NIV would be an advantage. McDermott et al. have recently presented the results of a randomised controlled trial (RCT) of diaphragm pacing in patients with MND, the Safety and Efficacy of Diaphragm Pacing in Patients with Respiratory Insufficiency Due to Amyotrophic Lateral Sclerosia (DiPALS) study [6]. Unfortunately, the trial had to be terminated prematurely because of a worse outcome in the paced group. The reasons for this are well discussed in their article [6] and an accompanying editorial [7]. A French trial [8] has also been stopped, following publication of the DiPALS study [6]; after a detailed, unplanned intermediate analysis, the sponsor decided to stop the study in the absence of benefits and because of a statistically significant excess mortality in the group of patients receiving active stimulation. In this study, patients were implanted at the time of diagnosis and received active or sham stimulation; patients, clinicians and investigators were

Received: Oct 162015 | Accepted: Oct 172015

Conflict of interest: Disclosures can be found alongside this article at openres.ersjournals.com

Copyright $\odot$ ERS 2015. This article is open access and distributed under the terms of the Creative Commons Attribution Non-Commercial Licence 4.0. 


\begin{tabular}{|c|c|c|}
\hline & Neurologist & Respiratory physician \\
\hline \multirow[t]{6}{*}{ History } & Breathlessness & Limb muscle weakness \\
\hline & Orthopnoea & Swallowing problems \\
\hline & Recurrent chest infections & Aspiration \\
\hline & Sleep quality & \\
\hline & Morning headaches & \\
\hline & Daytime sleepiness & \\
\hline \multirow[t]{4}{*}{ Examination } & Use of accessory muscles of respiration & Full neurological examination \\
\hline & $\begin{array}{l}\text { Reduced chest expansion on maximal } \\
\text { inspiration }\end{array}$ & $\begin{array}{l}\text { Muscle fasciculation labnormal muscle } \\
\text { movements may occur with }\end{array}$ \\
\hline & $\begin{array}{l}\text { Abdominal paradox linward movement } \\
\text { of the abdomen during inspiration } \\
\text { when supinel }\end{array}$ & hypercapnial \\
\hline & Ability to generate an adequate cough & \\
\hline \multirow[t]{4}{*}{ Investigations } & \multirow{4}{*}{\multicolumn{2}{|c|}{$\begin{array}{l}\text { Spirometry, mouth and sniff pressures, cough peak flow and oxygen saturation } \\
\text { should be performed at baseline and, thereafter, at three monthly intervals, } \\
\text { unless it is shown that the rate of decline allows a longer follow-up interval [3] } \\
\text { If the oxygen saturation is }<94 \% \text { or }<92 \% \text { in those with chronic lung disease, or if } \\
\text { they have sleep-related symptoms, arterial blood gas analysis and at a minimum } \\
\text { overnight oximetry with or without the addition of transcutaneous } \mathrm{CO}_{2} \text { or a } \\
\text { respiratory variable sleep study } \\
\text { Full polysomnography is not necessary } \\
\text { A raised } \mathrm{PaCO}_{2} \text { should lead to urgent specialist respiratory review (the patient } \\
\text { should be seen within no more than } 1 \text { week) [3]. }\end{array}$}} \\
\hline & & \\
\hline & & \\
\hline & & \\
\hline $\begin{array}{l}\text { Respiratory } \\
\text { physiotherapy }\end{array}$ & \multicolumn{2}{|c|}{$\begin{array}{l}\text { If the patients has weak cough, with a cough peak flow }<160 \mathrm{~L} \cdot \mathrm{min}^{-1}[4] \text {, or poor } \\
\text { glottic coordination because of bulbar involvement, there should be early review } \\
\text { by a respiratory physiotherapist with particular expertise in secretion clearance } \\
\text { techniques }\end{array}$} \\
\hline
\end{tabular}

all blinded to the intervention. If the preliminary results are confirmed following detailed analysis and peer review, they suggest that diaphragm stimulation per se is harmful, and that the worse outcome in the paced group is not due to an adverse effect of surgery and/or anaesthesia upon the natural history of MND. This is an important distinction, as the latter would have a bearing upon the advisability of other procedures, such as percutaneous gastrostomy. Similarly, it questions the advisability of diaphragm pacing in other situations; for instance, patients with critical care myopathy and difficulty weaning from assisted ventilation. At present, therefore, diaphragm pacing should not be considered in any patients with MND; its use in patients with other diagnoses should only be as part of a RCT.

Another device trial has recently been published [9] showing a worse outcome in patients receiving NIV, despite a robust physiological rationale for its use and a number of smaller uncontrolled studies suggesting benefit. Cheyne-Stokes respiration has been shown to be associated with a worse prognosis in patients with heart failure [10]; it can be successfully controlled using adaptive servoventilation (ASV), which improves sleep quality [11] and cardiac function [12]. However, despite improvements in quality of life, the mortality in patients with an ejection fraction $<45 \%$ was higher in the ASV group. It has been postulated that Cheyne-Stokes respiration may be protective [13] and the results of this trial provide some support for this hypothesis.

These trials raise wider questions for the NIV community and indeed for all clinicians using medical devices. The manufacturers of medical devices are not subject to the same stringency as the pharmaceutical industry when bringing new products to market. The double-blind, placebo-controlled trial is the gold standard for assessing any therapy, and no drug would be brought to the market without such evidence to underpin both efficacy and the absence of harm. This is not required for devices, which can become part of mainstream clinical practice without any published evidence at all, or only small physiological studies and/ or uncontrolled case series. However, a RCT is not without its problems. If a sham device is employed, it may be difficult to prevent the patient and/or clinician from realising whether they are receiving active or sham treatment. Furthermore, because many devices require technical expertise, which takes time to learn, by the time clinicians feel comfortable with them, they are already convinced that they "work". It is then very difficult for clinicians to maintain equipoise when it comes to recruitment into clinical trials. 
Clinicians will find an excuse not to randomise the patient that they are convinced will benefit from the intervention and as a result, there is the danger that individuals who are thought to be less likely to benefit get randomised and the trial has a negative result. However, the purpose of a clinical trial is to confirm not just benefit but also safety. The cautionary reminder from both the DiPALS and Adaptive Servo-Ventilation for Central Sleep Apnea in Systolic Heart Failure (SERVE-HF) studies is that unexpected harm can occur with devices, even those that have a beneficial physiological effect $[11,12,14]$.

So what is the answer to this problem? In the field of NIV, there are a number of situations in which the stable door cannot now be closed, as the horse has bolted. No clinician would be comfortable randomising patients with slowly progressive neuromuscular disease or chest wall deformity to a clinical trial in which a patient might be randomised to no intervention or just oxygen, with survival or quality of life as an end-point. The same is true for Duchenne muscular dystrophy, in which studies comparing with historical controls have shown a very substantial survival advantage $[15,16]$. There is, however, an issue around the introduction of new modes. There is nothing to stop manufacturers adding these to existing ventilator platforms and the clinician using them. While in theory, these are unlikely to do harm, the DiPALS study and SERVE-HF both show that this may not, in fact, be the case. Correction of a physiological abnormality may, surprisingly, cause harm. The application of devices to new groups of patients, as well as the use of new modes of ventilation in existing groups of patients, must be subjected to the same sort of scientific rigour as would be expected of a new drug. Small-scale, short-term, physiological studies should demonstrate a physiological benefit and an absence of immediate harm. These must then be followed by prospective RCTs with the clinical outcomes that are important to patients, ideally followed by post-marketing surveillance. This is likely to be challenging and have the effect of making device therapies more expensive because of the necessary investment in clinical trials, but will bring assurance to the clinical community that interventions are not just leading to significant benefits but also not causing harm.

There are two other interventions for patients with MND that are, or may, become standard care without robust evidence of benefit. These are the use of in/exsufflation devices (cough machines) and tracheostomy. Both are now widely used with evidence of an immediate effect, such that clinicians may be uncomfortable entering patients into RCTs. The potential harm of tracheostomy and the added burden that tracheostomy ventilation places upon carers are well documented. In/exsufflation is not without theoretical risks; high inflation pressures are used, with a resulting risk of pneumothorax. A small-scale RCT has suggested that in/exsufflation is not superior to breath stacking, paving the way for a RCT [17]. Where a RCT is not deemed possible, one answer would be comprehensive registries and an obligation to report adverse events, in the same way as is required for drugs. However, such registries are complex and expensive to set up, and there are questions about who should take responsibility for maintaining them, analysing the data and publishing the results. There may be a role here for professional societies.

In conclusion, respiratory physicians and neurologists should be cognisant of the respiratory complications of MND. NIV remains the mainstay of treatment with patients with sleep disordered breathing and respiratory failure due to MND. Diaphragm pacing should not be offered to any patient with MND. The results of the DiPALS and SERVE-HF trials should stimulate debate in the clinical and regulatory communities as to how device therapies should be best introduced into clinical practice. Laparoscopic diaphragm pacing for other conditions should only be performed as part of a RCT.

\section{References}

1 Bourke SC, Tomlinson M, Williams TL, et al. Effects of non-invasive ventilation on survival and quality of life in patients with amyotrophic lateral sclerosis: a randomised controlled trial. Lancet Neurol 2006; 5: 140-147.

2 Schiffman PL, Belsh JM. Pulmonary function at diagnosis of amyotrophic lateral sclerosis. Rate of deterioration. Chest 1993; 103: 508-513.

3 National Institute for Health and Clinical Excellence. Motor Neurone Disease: The Use of Non-Invasive Ventilation in the Management of Motor Neurone Disease. London, NICE, 2010.

4 Bach JR, Goncalves MR, Hamdani I, et al. Extubation of patients with neuromuscular weakness: a new management paradigm. Chest 2010; 137: 1033-1039.

5 Sancho J, Servera E, Morelot-Panzini C, et al. Non-invasive ventilation effectiveness and the effect of ventilatory mode on survival in ALS patients. Amyotroph Lateral Scler Frontotemporal Degener 2014; 15: 55-61.

6 Safety and efficacy of diaphragm pacing in patients with respiratory insufficiency due to amyotrophic lateral sclerosis (DiPALS): a multicentre, open-label, randomised controlled trial. Lancet Neurol 2015; 14: 883-892. Mitsumoto H. Non-invasive ventilation and diaphragmatic pacing in ALS. Lancet Neurol 2015; 14: 868-869.

8 Assistance Publique - Hôpitaux de Paris. Sclérose latérale amyotrophique (SLA) - maladie de Charcot: l'Assistance Publique-Hôpitaux de Paris décide de mettre un terme à l'étude clinique RespiStim [Assistance Publique - Hôpitaux de Paris stops the "RespiStimALS" clinical trial ("Early stage amyotrophic lateral sclerosis phrenic stimulation", NCT01583088)]. www.aphp.fr/contenu/sclerose-laterale-amyotrophique-sla-maladie-de-charcot-lassistance-publiquehopitaux-de Date last updated: August 24, 2015.

9 Cowie MR, Woehrle H, Wegscheider K, et al. Adaptive servo-ventilation for central sleep apnea in systolic heart failure. N Engl J Med 2015; 373: 1095-1105. 
Lanfranchi PA, Braghiroli A, Bosimini E, et al. Prognostic value of nocturnal Cheyne-Stokes respiration in chronic heart failure. Circulation 1999; 99: 1435-1440.

11 Teschler H, Dohring J, Wang YM, et al. Adaptive pressure support servo-ventilation: a novel treatment for Cheyne-Stokes respiration in heart failure. Am J Respir Crit Care Med 2001; 164: 614-619.

12 Arzt M, Schroll S, Series F, et al. Auto-servoventilation in heart failure with sleep apnoea: a randomised controlled trial. Eur Respir J 2013; 42: 1244-1254.

13 Naughton MT. Cheyne-Stokes respiration: friend or foe? Thorax 2012; 67: 357-360.

14 Gonzalez-Bermejo J, Morelot-Panzini C, Salachas F, et al. Diaphragm pacing improves sleep in patients with amyotrophic lateral sclerosis. Amyotroph Lateral Scler 2012; 13: 44-54.

15 Simonds AK, Muntoni F, Heather S, et al. Impact of nasal ventilation on survival in hypercapnic Duchenne muscular dystrophy. Thorax 1998; 53: 949-952.

16 Eagle M, Baudouin SV, Chandler C, et al. Survival in Duchenne muscular dystrophy: improvements in life expectancy since 1967 and the impact of home nocturnal ventilation. Neuro muscul Disord 2002; 12: $926-929$.

17 Rafiq MK, Bradburn M, Proctor AR, et al. A preliminary randomized trial of the mechanical insufflator-exsufflator versus breath-stacking technique in patients with amyotrophic lateral sclerosis. Amyotroph Lateral Scler Frontotemporal Degener 2015: 1-8. 